\title{
HMPG secures top-drawer CEO
}

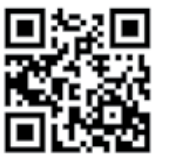

The Health and Medical Publishing Group (HMPG), owned by the South African Medical Association (SAMA), has scored a coup in securing Hannah Kikaya, a former senior editor at The Lancet and a top South African (SA) health systems and communications strategist, as its new CEO.

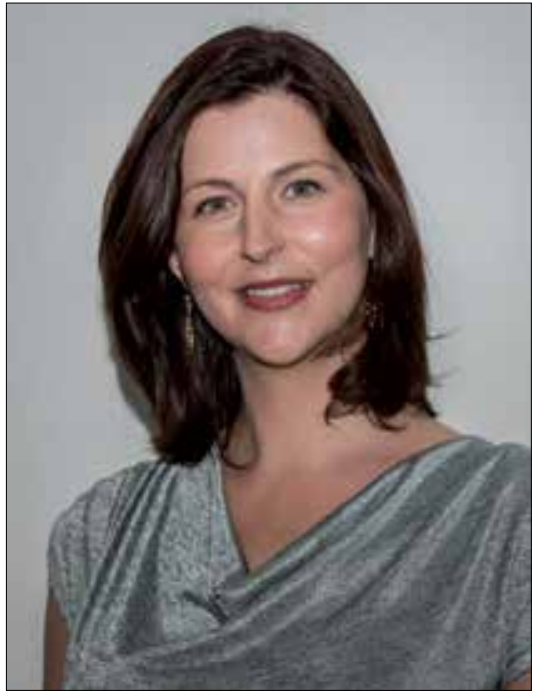

Hannah Kikaya, HMPG's new CEO.

She will succeed Gert Steyn, the new SAMA General Manager, who over the past two years, both before and during his current tenure, revitalised HMPG, the country's leading medical publishing group, by taking it from a net loss-making enterprise to a thriving, financially sound, technologically relevant and income-diverse platform. Kikaya will use this springboard to apply her unique combination of academic publishing experience, global policy development and practical implementation skills (seemingly tailor-made for her new job). With 14 years' experience in public health, she has specialised in multisectoral publicprivate relationship building in challenging environments, been intimately involved in helping lay the groundwork for SA's national health system reforms, co-ordinated regional country planning to discuss tuberculosis (TB) in the mining sector, and organised community participation in health systemsstrengthening initiatives. She is also the inaugural editor of HMPG's latest journal title, Strengthening Health Systems, an exciting new international open-access journal and publishing support resource for developing countries, launched in July last year.

\section{Bringing rare hands- on experience to our journals}

Kikaya will bring rare hands-on experience to shaping the texture and content of HMPG's 12 journals, of which SAMJ is the flagship. While a consultant to the World Bank (2011 - 2013) and the Stop TB Partnership (World Health Organization and Global Business Coalition, Health (SA)) (2013/14), she designed and successfully implemented a revenue and administrative efficiency-boosting programme for Gauteng's main referral hospitals and supported the drafting of the Southern African Development Community (SADC) Declaration on TB in the Mining Sector. She provided technical guidance for a regional project to establish minimum standards for national policies on the management of paediatric HIV, TB and malaria treatment protocols in SADC member states, and finalised a mid-term review and various donor reports for the Lesotho Country Office of UNICEF. She has more than 50 publications in scientific journals, with a further 50 editorial contributions to The Lancet (most as the main leader writer). Her publishing and media experience include a year as part-time Managing Editor of Molecular Oncology (2008/9), a 21/2-year stint as Senior Editor of Lancet Oncology (2001 - 2003), Editor at Current Drugs Limited (1999 - 2000), and speech writer and researcher for Ian Taylor, an MP in the British House of Commons (part of a 4-month internship in 1999).

Fluent in French and English and holder of dual South African-British citizenship, she holds a BSc with joint honors in Physiology and Pharmacology from University College London.

\section{HMPG 2020}

Kikaya's vision for HMPG is to put the needs of SA's scientists and health practitioners at centre stage by transforming the company into a diverse provider of publishing and educational services to support academic enquiry, knowledge exchange and professional development across the region.

She explains that academic publishing was first established to facilitate the exchange of new knowledge between scientists and investigators, usually in the context of learned societies. However, as the field developed and commercial incentives took hold, the money required for scientists to be able to communicate their work, and the subscription costs for others to read it, led to a situation where knowledge exchange was effectively hindered rather than supported by the publication process especially for researchers in low-resource environments.

Under Kikaya, who recognises the company's strong history of supporting academic societies and research in South Africa, HMPG will re-emphasise its commitment to open-access publication of all journal content and pledges to maintain its much-lauded policy of no author fees for submissions.

'I strongly believe that the free exchange of knowledge among academics and health and medical practitioners is crucial to the betterment of public health, both in this country and worldwide. However, achieving publication in established academic journals is still a significant challenge for many of SA's aspiring scientists and practitionerinvestigators. These publication barriers hinder the kinds of knowledge exchange that could catalyse rapid advancements in health and medicine. We at HMPG pledge to support the growth of academic enquiry and scientific publication in this country and find innovative solutions to professional learning that promote, rather than hinder, the generation and use of new knowledge, says Kikaya.

Key to her five-year strategy is to bring the company up to date with the internet age. Online publishing presents a huge opportunity for sharing of information among academic communities, but technological challenges and infrastructure weaknesses have so far prevented academics from reaping the full benefits of these developments. Understanding the crucial role of the internet for current and future knowledge exchange, Kikaya has pledged to give HMPG's online publishing interface and web-based library the prominence it deserves under her leadership.

'We want to ensure that all academic investigators, no matter how big or small their research grant, or how powerful or weak their institution, can share and access the knowledge that helps move the country forward to better health. It is as simple as that,' she says.

\section{Chris Bateman}

chrisb@hmpg.co.za

S Afr Med J 2015;105(2):82.

DOI:10.7196/SAMJ.9383 